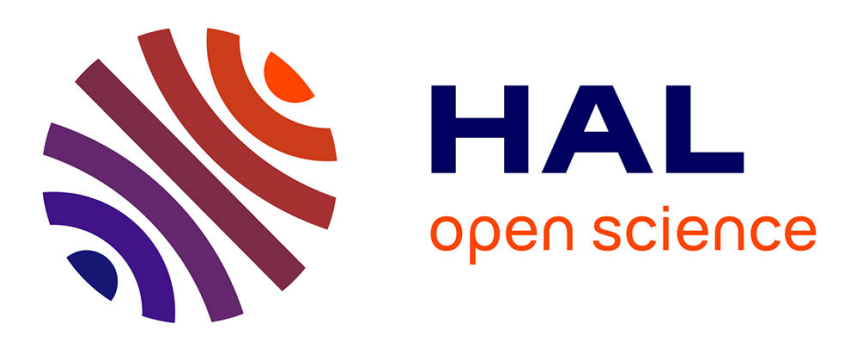

\title{
Numerical Investigation of a Mistuned Academic Bladed Disk Dynamics with Blade/Casing Contact
}

\author{
Jeanne Joachim, Florence Nyssen, Alain Batailly
}

\section{To cite this version:}

Jeanne Joachim, Florence Nyssen, Alain Batailly. Numerical Investigation of a Mistuned Academic Bladed Disk Dynamics with Blade/Casing Contact. Journal of Engineering for Gas Turbines and Power, 2020, 10.1115/1.4047780 . hal-02896893

\section{HAL Id: hal-02896893 https://hal.science/hal-02896893}

Submitted on 11 Jul 2020

HAL is a multi-disciplinary open access archive for the deposit and dissemination of scientific research documents, whether they are published or not. The documents may come from teaching and research institutions in France or abroad, or from public or private research centers.
L'archive ouverte pluridisciplinaire HAL, est destinée au dépôt et à la diffusion de documents scientifiques de niveau recherche, publiés ou non, émanant des établissements d'enseignement et de recherche français ou étrangers, des laboratoires publics ou privés. 


\title{
Numerical Investigation of a Mistuned Academic Bladed Disk Dynamics with Blade/Casing Contact
}

\author{
Jeanne Joachim ${ }^{1}$, Florence Nyssen ${ }^{1}$, Alain Batailly ${ }^{1}$
}

\begin{abstract}
This contribution focuses on the combined analysis of mistuning and unilateral blade-tip/casing contacts. A 2D phenomenological finite element model of an aircraft engine fan stage is considered. It is reduced by means of the Craig-Bampton component mode synthesis method and contact treatment relies on a Lagrange multiplier algorithm within an explicit time-integration scheme. Blade-tip/casing contacts are initiated through the deformed shape of a perfectly rigid casing. Mistuning is accounted for on the blades only. Monte Carlo simulations are carried out in both linear and nonlinear configurations, which allows to compare amplifications predicted in both context due to mistuning. Following a thorough convergence analysis of the proposed numerical strategy, the influence of mistuning level as well as the configuration of the external forcing are investigated. Presented results underline the detrimental consequences of mistuning in a nonlinear structural context, yielding even higher vibration amplifications than in a linear context. A cross-analysis between linear and nonlinear computations reveals that no correlation is found between linear and nonlinear amplifications which suggests that the effect of existing strategies to mitigate vibration amplifications within a linear context may not be suitable within a nonlinear context.
\end{abstract}

Keywords

mistuning; nonlinear dynamics; rotor/stator interaction; stochastic dynamics; unilateral contact constraints

1 - Department of Mechanical Engineering, École Polytechnique de Montréal, P.O. Box 6079, Succ. Centre-Ville, Montréal, Québec, Canada H3C 3A7 


\title{
Analyse du comportement vibratoire d'un modèle numérique de roue aubagée désaccordée avec interfaces de contact aubes/carter
}

\author{
Jeanne Joachim ${ }^{1}$, Florence Nyssen ${ }^{1}$, Alain Batailly ${ }^{1}$
}

Résumé

Cet article présente une analyse combinée du désaccordage et du contact aube/carter pour un modèle de roue aubagée phénoménologique en deux dimensions. Ce modèle éléments finis est réduit à l'aide d'une méthode de synthèse modale et le traitement du contact est opéré via un algorithme reposant sur le calcul de multiplicateurs de Lagrange au sein d'une procédure d'intégration temporelle de type différences finies centrées. Le contact aube/carter est initié par une déformation du carter supposé parfaitement rigide. Le désaccordage est pris en compte uniquement au niveau des aubes et des simulations de type Monte Carlo sont effectuées dans un contexte linéaire (réponse forcée à un chargement aérodynamique simplifié) et non-linéalire (réponse vibratoire au contact). Une attention particulière est portée sur la validation des résultats obtenus en s'assurant notamment de la convergence stochastique, par des indicateurs quantitatif et qualitatif. Les résultats obtenus attestent de la représentativité du modèle étudié par rapport aux standards de la littérature dans un contexte linéaire. Par ailleurs, lorsque le contact aubes/carter est pris en compte, les résultats obtenus montrent une augmentation significative des amplifications de vibration par rapport à celles observées dans un contexte linéaire. Une analyse croisée des résultats linéaire et non-linéaire souligne l'absence de corrélation entre les motifs engendrant les plus grandes amplifications linéaire et non-linéaire. Cette dernière observation suggère que les méthodes existantes pour réduire l'amplification des vibrations dans un contexte linéaire pourraient ne pas être adaptées à la réduction des amplifications dans le cas de contacts aubes/carter.

Mots-clés

désaccordage; dynamique non-linéaire; interactions rotor/stator; dynamique stochastique; contact unilatéral

1 - Département de génie mécanique, École Polytechnique de Montréal, P.O. Box 6079, Succ. Centre-Ville, Montréal, Québec, Canada H3C 3A7 


\section{Introduction}

Many research efforts have been dedicated to the analysis of bladed disk mistuning [1], a phenomenon that characterizes the unavoidably non-perfectly symmetric shape of aircraft engine rotating components, be it due to manufacturing tolerances or material inhomogeneities. Contrary to perfectly symmetric structures, mistuned bladed disks feature frequency split [2] that impact their dynamic properties. Ever since the identification of localization phenomena [3,4] related to mistuning and their detrimental effects due to high cycle fatigue [1], industry-driven research has focused on mitigating mistuning undesired consequences on bladed components' forced response. Within this linear framework, a wide variety of solutions have been investigated such as the development of novel blade designs $[5,6,7]$ and intentional mistuning patterns with optimized blade arrangements $[8,9,10]$. Very recently, researchers started to account for mistuning within nonlinear contexts such as the analysis of flutter interactions [11,12] or blade root friction damping issues [13]. These studies suggest that intentional mistuning can even be used as a way to reduce bladed disk vibration amplitudes, hence improving modern aircraft engine designs. Yet, the robustness of intentionally mistuned blade designs should still be assessed with regards to mistuning. In both linear and nonlinear contexts, robust numerical predictions accounting for a bladed disk mistuning are typically achieved by means of Monte Carlo simulations [14] or polynomial chaos expansion [15].

Nonlinear vibrations subsequent to blade/casing structural contacts are among the nonlinear phenomena of interest for aircraft engine manufacturers [16]. As a matter of fact, the drive towards increased overall engine efficiency motivates engineers to reduce operating clearances [17] to a point where blade/casing contacts must now be accounted for at the design stage. The damaging consequences of rubbing events-contacts between a blade and the surrounding abradable liner deposited on the casing contact surface-have been evidenced both experimentally [18] and numerically [19]. The development of efficient numerical strategies now allows the vibration analysis of full bladed disks undergoing structural contacts [20, 21, 22]. In order to ensure reliable simulation results, it is important to assess the robustness of numerical predictions with respect to a certain level of mistuning. The intrinsically nonlinear nature of contact simulations calls for thorough analyses as mistuning may potentially broaden the scope of investigation by altering the bladed disk dynamics or yielding unexpected co-existing solutions.

To the best of the authors' knowledge, this paper presents the first analysis of the influence of small mistuning (also referred to as natural mistuning in the literature) on a bladed disk nonlinear response following bladetips/casing unilateral contacts. Stochastic analyses are conducted based on well-established methods for contact treatment and mistuning implementation applied on a 2D phenomenological finite element (FE) model of a civil aircraft engine fan stage [23]. Material parameters and mechanical properties of the model are adjusted in order to obtain physically relevant frequencies for the first blade bending modes. Time integration procedures are here preferred to frequency solution methods. While this choice implies longer computation times, it yields a methodology that does not rely on any assumption with regard to the nature of the solution: periodic, quasiperiodic or chaotic motions may thus be predicted. It also provides insight on transient phenomena which have been identified as critical for the analysis of contact initiated interactions [22, 24]. A thorough validation procedure is employed in order to ensure that presented results are numerically relevant. As robust and converged nonlinear forced response functions are essential for the definition of nonlinear amplification, the stabilized permanent regimes are assessed by means of an autocorrelation technique. With respect to contact treatment, a Lagrange multiplier based approach [25] is preferred to penalty methods [26, 13] in order to avoid any inter-penetrations between the bladed disk and the surrounding casing. This contact treatment algorithm has been successfully confronted to alternated numerical approaches in [27]. Finally, mistuning of the bladed disk is limited to the blades $[28,1]$ and accounted for through a variation of each blade Young's modulus.

In the second section of the article, the considered phenomenological model is presented in details, along with the stochastic characterization of the associated mistuned system in a linear context. The third section then focuses on the presentation and validation of the proposed methodology for nonlinear contact simulations on a given mistuned bladed disk. In the fourth section, a nonlinear stochastic analysis is carried out: convergence of the stochastic simulations is assessed and nonlinear amplifications are introduced.

The influence of structural damping on nonlinear amplifications is also discussed. Finally, the last section of 
Table 1. Geometrical parameters and material properties of the finite element model

\begin{aligned} \hline number of blades & $N=12 \\$\hline tip radius & $50 \mathrm{~cm} \\$\hline hub radius & $20 \mathrm{~cm} \\$\hline nominal Young's modulus & $E_{0}=2.1 \cdot 10^{11} \mathrm{~Pa} \\$\hline density & $7800 \mathrm{~kg} / \mathrm{m}^{3} \\$\hline modad damping ratio & $5 \cdot 10^{-3} \\$\hline Poisson's ratio & 0.3\end{aligned}

this paper provides an analysis of identified co-existing stable solutions.

\section{Modeling}

The 2D phenomenological FE model used in this study is depicted in Fig. 1a. The blades are discretized with straight beam elements with 3 degrees of freedom (dof) per node whereas the disk is made of both straight and curved beam elements featuring 4 dof per node. The model dimensions and properties, summarized in Tab. 1, are adjusted so that the first bending modes frequencies are in the vicinity of $90 \mathrm{~Hz}$. This model is similar to the one used in previous publications dedicated to a variety of contact phenomena for a tuned bladed disk [23, 29] where it was found to be fairly representative of an actual aircraft engine fan stage. It should also be underlined that the proposed methodology, though applied to a 2D model, is inherently applicable to 3D industrial models.

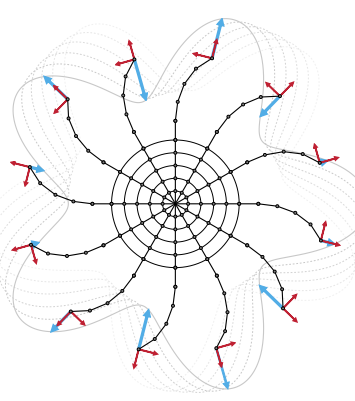

(a) linear configuration $\left(n_{d}=5\right)$ : circular load $(\longrightarrow)$ and boundary $\operatorname{dof}(\longrightarrow)$

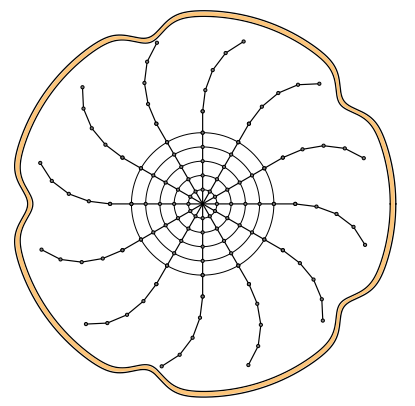

(b) nonlinear configuration $\left(n_{d}=5\right)$ : deformed casing $(\square)$

Figure 1. 2D bladed disk model

\subsection{Model Reduction}

The Craig-Bampton component mode synthesis method [30] is used to reduce the dimension of the model and thus lower its computational cost. This reduction technique advantageously yields a mixed reduced set of coordinates in which physical dof can be kept. This way, displacements in both directions of space at the tip of each of the 12 blades are retained within the reduced order model (ROM) for efficient contact treatment. These 24 boundary dof, see Fig. 1a, are complemented by $\eta$ modal dof. The SAFE diagram for both the ROM and the full-FE model is 


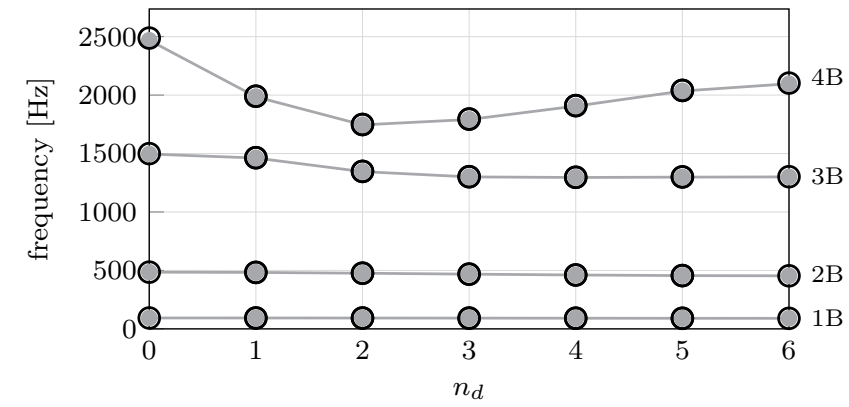

Figure 2. SAFE diagram for the ROM with $\eta=60(\mathrm{O})$ and for the full-FE model $(-\bullet)$

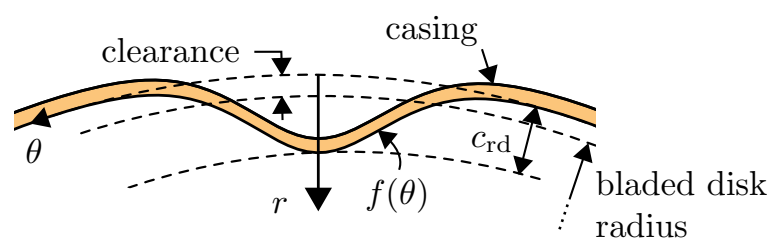

Figure 3. Casing deformation

depicted in Fig. 2. It can be seen that modes of the first bending mode (1B) are closely spaced in frequency, from $90.1 \mathrm{~Hz}$ to $93.2 \mathrm{~Hz}$.

\subsection{Contact Treatment}

\subsubsection{Contact Initiation}

In this study, contact is initiated by a deformation of the surrounding rigid casing, see Fig. $1 \mathrm{~b}$ and Fig. 3. For the sake of simplicity, the number of privileged contact areas on the casing is here defined as $n_{d}$, in analogy with the number of nodal diameters of free vibration modes that would be excited by such configuration for the tuned system. Each of the $n_{d}$ casing bumps are evenly spaced along the casing circumference. A smooth evolution of the contact surface is achieved through an exponential growth of the bumps in the first $0.01 \mathrm{~s}$ of the simulation in order to avoid any initial blade/casing penetration. The variation of the casing radius due to these bumps is referred to as the casing radial deformation amplitude $c_{\mathrm{rd}}$. Each bump is defined using the function:

$f(\theta)=-c_{\mathrm{rd}} \exp \left(-\left(\frac{\theta}{0.1 \frac{2 \pi}{n_{d}}}\right)^{2}\right)$

with $\theta$ the angular position. As depicted in Fig. 3, contact may occur when $c_{\text {rd }}$ is higher than the initial blade/casing clearance.

\subsubsection{Numerical Solution}

Within the small perturbation framework, the equation of motion to be solved in the time domain is:

$\mathbf{M} \ddot{\mathbf{u}}+\mathbf{D} \dot{\mathbf{u}}+\mathbf{K u}=\mathbf{F}(t)+\mathbf{F}_{\mathrm{nl}}(\mathbf{u}, t)$

where $\mathbf{M}, \mathbf{D}$ and $\mathbf{K}$ are respectively the mass, damping and stiffness matrices, $\mathbf{u}$ denotes the generalized displacements vectors, $\dot{\mathbf{u}}$ and $\ddot{\mathbf{u}}$ respectively their first and second order time derivatives, $\mathbf{F}$ and $\mathbf{F}_{\mathrm{nl}}$ are the linear and nonlinear excitation force vectors. The forward increment Lagrange multiplier algorithm [25] is employed to compute the solution of Eq. (2). The associated prediction/correction algorithm is used similarly to what was previously described in [23]. 


\subsection{Mistuning}

\subsubsection{Implementation}

Small mistuning is implemented through material properties variations, thus echoing with tolerances formulation of industrial manufacturing processes. As blade mistuning has been shown to be critically dominant $[28,1]$, mistuning is here considered as a blade Young's modulus variation:

$E_{i}=E_{0}\left(1+(\delta E)_{i}\right) \quad, \quad i \in[1, N]$

with $E_{i}$ the Young's modulus for the blade $i, N$ the number of blades and $E_{0}$ the nominal Young's modulus of the tuned system. The Young's modulus variation $(\delta E)_{i}$ is randomly drawn for each system in a range $[-d E,+d E]$, assuming a uniform distribution. The normalized standard deviation of random mistuning, used to draw the Monte Carlo simulations results [14], is thus given by:

$\sigma(d E)=\frac{2 d E}{\sqrt{12}}$

The variation $d E$ is set up to $4 \%$, so that $\sigma(d E)$ varies up to $2.3 \%$, in agreement with levels of mistuning identified for modern manufacturing processes [31]. In the remainder, $\sigma(d E)$ is called the mistuning level and is denoted $\sigma$ for the sake of brevity.

\subsubsection{Linear Amplification}

Tuned and mistuned bladed disks are excited by a circular load of amplitude $F_{\text {lin }}\left(\theta_{i}, t\right)$ and applied in the radial direction, see Fig. 1a. This load is a simplified representation of an aerodynamic forcing. Its application is limited to boundary dof in order to avoid any numerical artifact related to the modal reduction procedure:

$F\left(\theta_{i}, t\right)=F_{\max } \cos \left(\left(\theta_{i}-\Omega t\right) n_{d}\right)$

where $\theta_{i}$ stands for the angular position of the $i^{\text {th }}$-blade, $\Omega$ is the angular speed, and $F_{\max }$ is the amplitude of the forcing. It is here set to $F_{\max }=150 \mathrm{~N}$. Finally, $n_{d}$ represents the space frequency of this loading and is related to the number of nodal diameters of the excitation. By choice, this notation is identical to the one used for the nonlinear excitation for the sake of later comparisons.

For each analyzed mistuned bladed disk, only the maximal vibration amplitude is retained over the frequency range of interest [14]. The ratio between the maximal vibration amplitude of the mistuned model and the one of the tuned model, classically called the mistuning amplification, will be referred to in the linear context as the linear amplification $A_{\text {lin }}$.

Characterization of a bladed disk sensitivity to mistuning requires a stochastic analysis with a large number of samples, each corresponding to a specific mistuned configuration. Stochastic convergence, defined by the stability of the percentile values with the number of samples, is typically achieved with several thousands of samples [1]. For the proposed 2D bladed disk model, obtained results for the first bending modal family (1B) are depicted in Fig. 4 for $n_{d}=1, \ldots, 6$ and $\sigma \in[0,2.3] \%$. The stochastic convergence has been observed starting from 10,000 samples at each point $\left(n_{d}, \sigma\right)$ of the parameter space thus requiring a total of 240,000 samples. Three quantities are plotted for each point $\left(n_{d}, \sigma\right)$ corresponding to percentile 1, percentile 50 and percentile 99 of the amplification $A_{\text {lin }}$.

Monte Carlo simulations carried out for various number of $n_{d}$, depicted in Fig. 4 , provide insight on the sensitivity of $A_{\text {lin }}$ with respect to $n_{d}$. $A_{\text {lin }}$ is maximal for $n_{d}=6$ and minimal for $n_{d}=3$, and does not vary monotonously with $n_{d}$ which is consistent with the literature [32]. The general shape of $A_{\text {lin }}$ with regards to $n_{d}$ is in good agreement with previous findings for the 12-blade model used in [33]. Also, the order of magnitude for the amplification in a linear context are similar to typical values found in the literature [34, 14].

\section{Nonlinear Analysis of a Mistuned Bladed Disk}

In order to illustrate the proposed methodology, a random mistuning pattern with mistuning level $\sigma=1.7 \%$ is chosen, see Tab. 2. Key parameters and quantities of interest which are used for the stochastic analysis in the next 


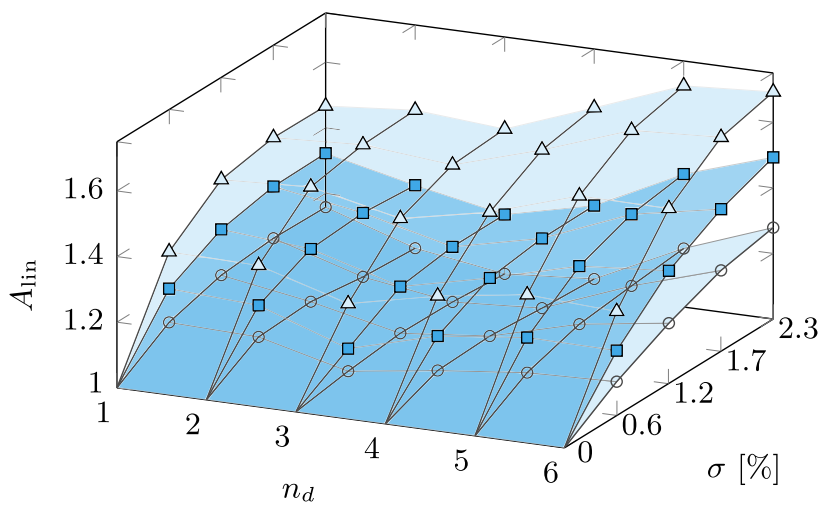

Figure 4. Predicted amplifications for the first bending modal family: percentile 1 ( $\multimap-$ ) , percentile 50 ( $\square-)$ and percentile $99(-\triangle-)$

Table 2. Young's modulus per blade $\left(\times 10^{11} \mathrm{~Pa}\right)$ for the chosen random mistuning pattern

\begin{tabular}{llllll}
\hline$E_{1}$ & 2.0701 & $E_{2}$ & 2.0627 & $E_{3}$ & 2.0695 \\
\hline$E_{4}$ & 2.1683 & $E_{5}$ & 2.0809 & $E_{6}$ & 2.1421 \\
\hline$E_{7}$ & 2.1164 & $E_{8}$ & 2.0166 & $E_{9}$ & 2.1256 \\
\hline$E_{10}$ & 2.0328 & $E_{11}$ & 2.0391 & $E_{12}$ & 2.1193 \\
\hline
\end{tabular}

section are presented. Because the validation of the overall numerical procedure related to contact management was previously published [23], particular attention is here paid to: (1) the convergence of the simulations with regards to the 2D bladed disk reduction parameter $\eta$ and the time step $h$ and, (2) the robustness of the obtained results with respect to the different parameters of the model.

\subsection{Steady State Detection}

Contact simulations are carried out assuming a constant angular speed and considering a deformed casing as depicted in Fig. 1b. To ensure that a steady state has been reached, time responses are thoroughly checked by means of an autocorrelation technique, with a focus on the tangential displacement of the blade-tip featuring the highest vibratory amplitudes. Simulations are first run over $0.5 \mathrm{~s}$, then the time response over the last three revolutions is compared to the time response over the previous three revolutions, see Fig. 5. The correlation between these two signals is evaluated through the Pearson product-moment correlation coefficient (PPMCC). The steady state is considered to be reached when two criteria are satisfied: (1) the PPMCC must be higher than the threshold value $c_{\text {cor }}$ and, (2) the relative error on the maximal amplitude must be lower than $\epsilon$. If any of these two criteria is not satisfied, the simulation is run over an additional ten revolutions until a steady state has been reached. In this study, a good compromise between accuracy and computation times was obtained by considering $c_{\text {cor }}=95 \%$ and $\epsilon=1 \%$. On average, steady states are reached for $t=0.96 \mathrm{~s}$.

\subsection{Interaction Maps}

Contact simulations are first performed over a wide angular speed range (from $10 \mathrm{~Hz}$ to $80 \mathrm{~Hz}$ ) on both the tuned and the mistuned bladed disk, for $n_{d}=4$, in order to better understand their vibration behavior. The Fourier transform of the time responses obtained for each angular speed is computed to generate the interaction maps, shown in Fig. 6, where darker areas account for higher amplitudes.

For the tuned bladed disk, the highest amplitudes of vibration in Fig. 6a are witnessed for angular speeds in the vicinity of $34.45 \mathrm{~Hz}$ and $69 \mathrm{~Hz}$. These interactions respectively correspond to the crossing between the first 


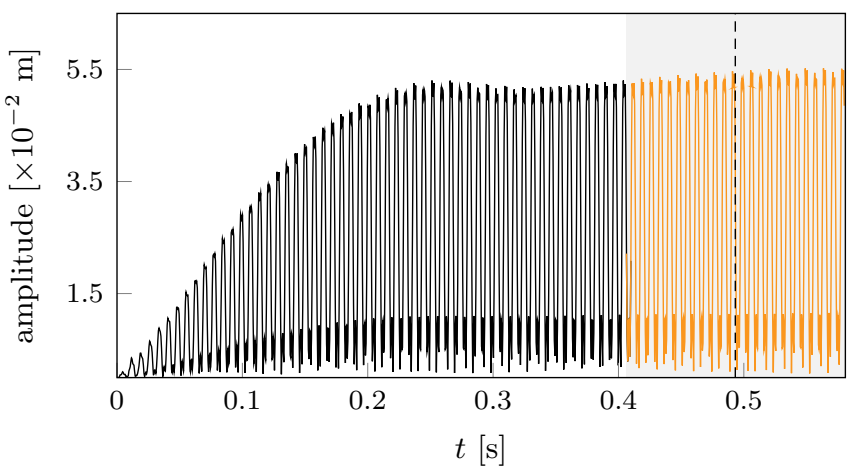

(a) full time response

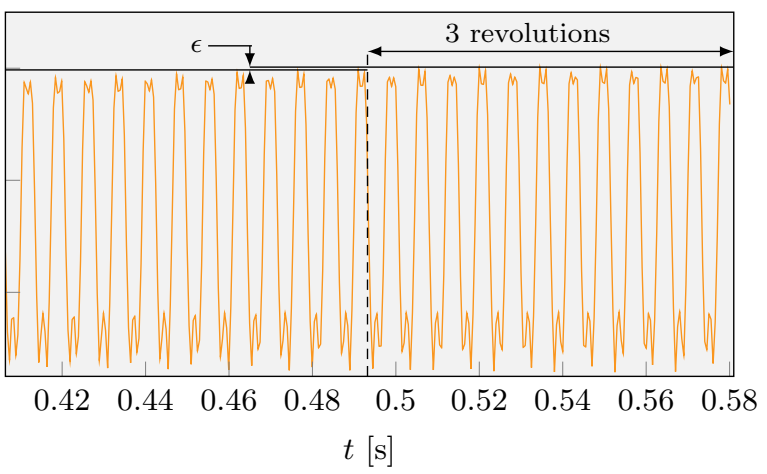

(b) zoom over the last 6 revolutions

Figure 5. Time response obtained for $\Omega=34.45 \mathrm{~Hz}$ and $n_{d}=4$, with area of interest for steady state detection ( - )

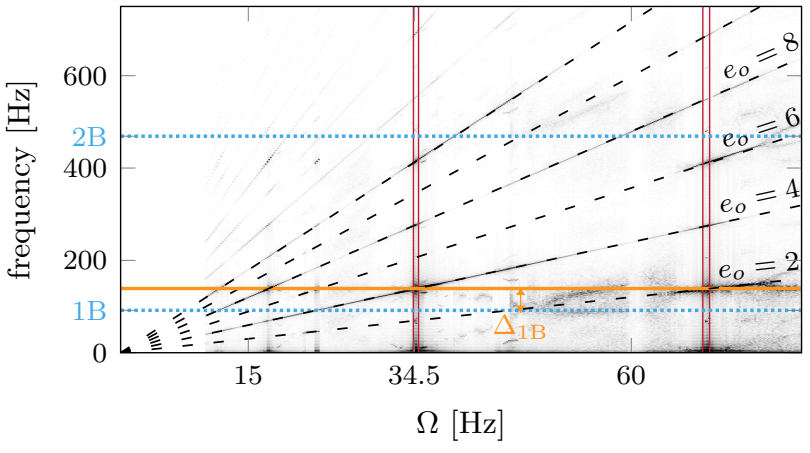

(a) tuned bladed disk

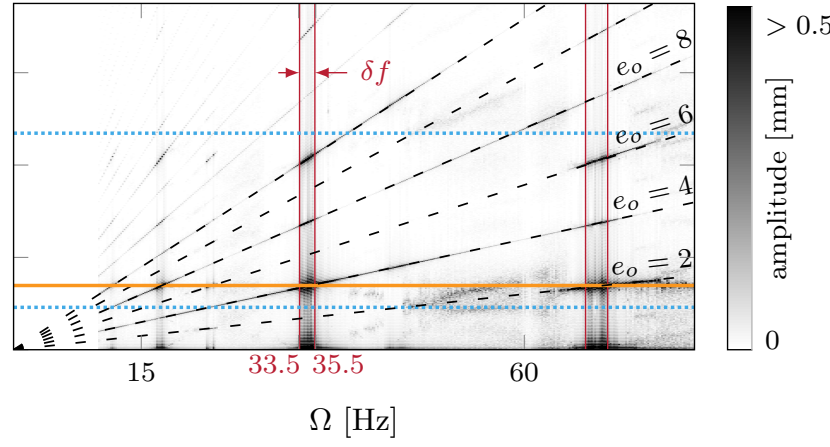

(b) mistuned bladed disk

Figure 6. Interaction maps for $n_{d}=4$, with engine order lines ( - - ), linear natural frequencies ( $=$ - ), nonlinear frequencies $(-$ ) and interaction areas $(-)$ with the highest amplitudes 
Table 3. Angular speed range of interest for the mistuned bladed disk

\begin{tabular}{lcccc}
\hline$n_{d}$ & 3 & 4 & 5 & 6 \\
\hline$\Omega_{\min }[\mathrm{Hz}]$ & 44.5 & 33.5 & 26.7 & 22 \\
\hline$\Omega_{\max }[\mathrm{Hz}]$ & 47.5 & 35.5 & 28.2 & 23 \\
\hline
\end{tabular}

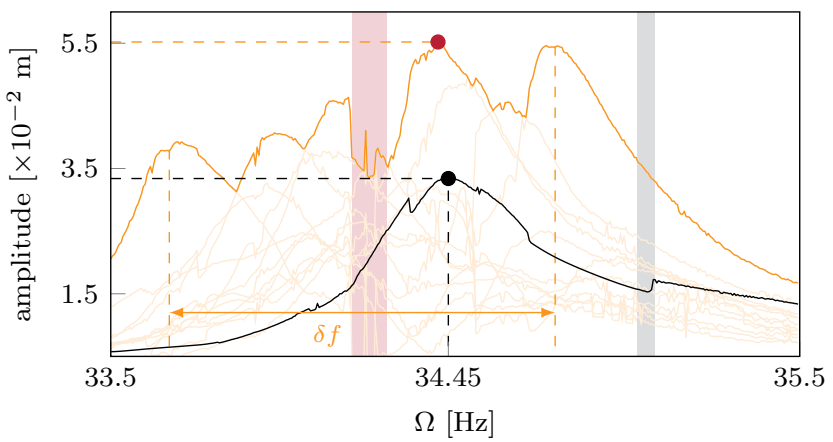

Figure 7. FRF for $n_{d}=4$ : mistuned $(-)$ and tuned bladed disk $(\boldsymbol{-})$

bending modal family (1B) of the bladed disk and the fourth $\left(e_{o}=4\right)$ and second $\left(e_{o}=2\right)$ engine orders. It is visible that interactions are actually predicted for frequencies slightly higher (see $\Delta_{1 \mathrm{~B}}$ in Fig. 6a) than those of the first bending modal family which is due to the contact stiffening phenomenon [21].

The interaction maps, as depicted in Fig. $6 \mathrm{~b}$ for the mistuned bladed disk, are key to define angular speed ranges of interest for the rest of this study. The influence of mistuning is noticeable, with both interaction areas spreading in a wider range $\delta f$ due to the frequency split phenomenon. Hence, the width of the angular speed range to consider to capture the maximal amplitudes of vibration depends on the mistuning pattern. Besides, for different values of $n_{d}$ different interaction speeds are predicted. Stochastic analyses on the samples presented in the next section led to the angular speed range summed up in Tab. 3, with a focus on the crossing between the 1B frequency line and the engine order $e_{o}=n_{d}$.

\subsection{Nonlinear Forced Response Function}

Based on the angular speed ranges previously identified, contact simulations are run with an angular speed increment $\delta \Omega=0.05 \mathrm{~Hz}$. For each angular speed, the amplitude of the steady state is retrieved to draw the nonlinear forced response function (FRF) of the bladed disk. As an example, the FRF of both the mistuned bladed disk and the tuned bladed disk for $n_{d}=4$ are pictured in Fig. 7. Here again, it is noticeable that the frequency spreading $\delta f$ induced by mistuning significantly increases the angular speed range over which high amplitudes of vibration are found.

For each considered mistuning pattern, the quantity of interest is the maximal amplitude of vibration over the angular speed range [14]. The division of this quantity by the maximal amplitude of vibration of tuned bladed disk, found for $\Omega=34.45 \mathrm{~Hz}$, yields the nonlinear amplification, denoted $A_{\mathrm{nl}}$ in the following.

The intrinsically nonlinear nature of the investigated mechanical system requires that particular attention be paid to the obtained FRF in order to assess the robustness of the procedure. Indeed, it is likely that, due to unilateral contact constraints, the system exhibits an intricate dynamics featuring a variety of stable branches and bifurcations [35], as have already been observed for 3D finite element models in similar contact configurations [36]. The FRF pictured in Fig. 7 does show signs of such occurrences, see, for instance, the sudden changes in amplitudes of vibration for the mistuned model (red area) and the tuned model (gray area). Yet a careful analysis of these jumps for various mistuning patterns reveals that they are not located in the vicinity of a peak and thus it is assumed that they do not impact the detection of the maximal of amplitude. These changes in amplitudes are further analyzed 


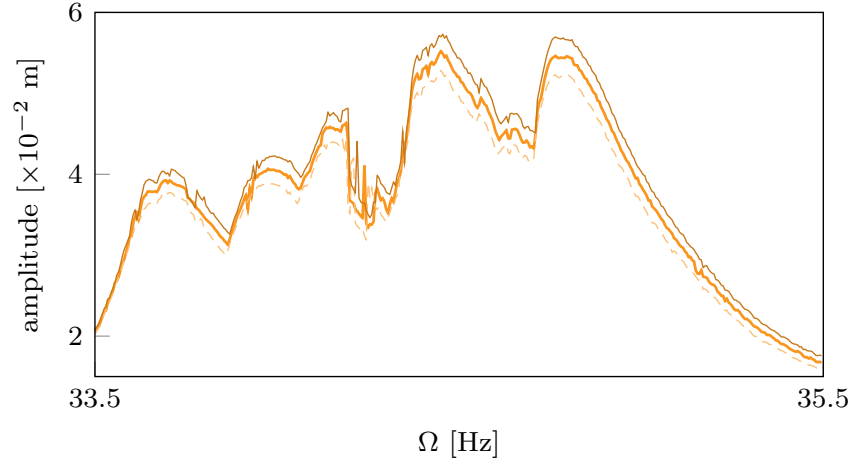

(a) varying casing deformation amplitude: $1.20 \mathrm{~mm}(==)$, $1.25 \mathrm{~mm}(-)$ and $1.30 \mathrm{~mm}(-)$

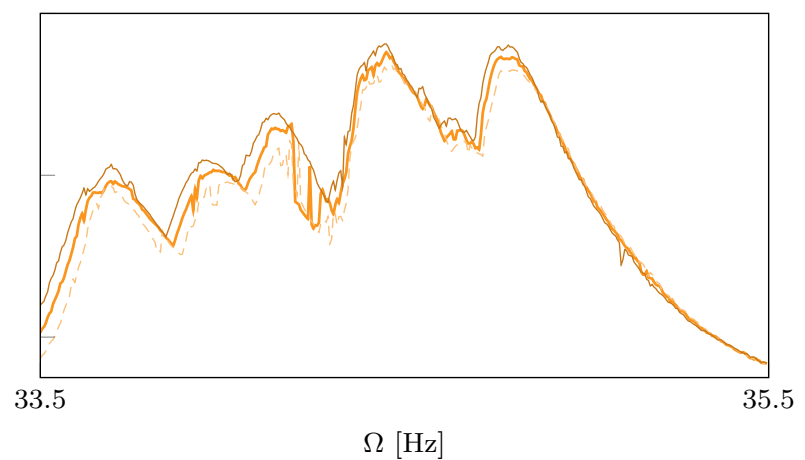

(b) varying initial clearance: $0.20 \mathrm{~mm}(==)$, $0.25 \mathrm{~mm}(-)$ and $0.30 \mathrm{~mm}(-)$

Figure 8. FRF for varying casing parameters

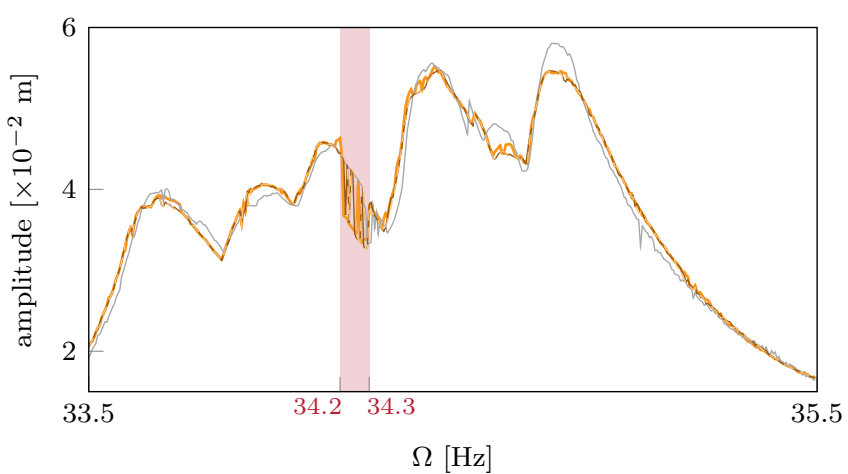

(a) time convergence: $h=5 \times 10^{-5} \mathrm{~s}(-), h=5 \times 10^{-6} \mathrm{~s}$ $(--), h=10^{-6} \mathrm{~s}(-)$ and $h=5 \times 10^{-7} \mathrm{~s}(-)$

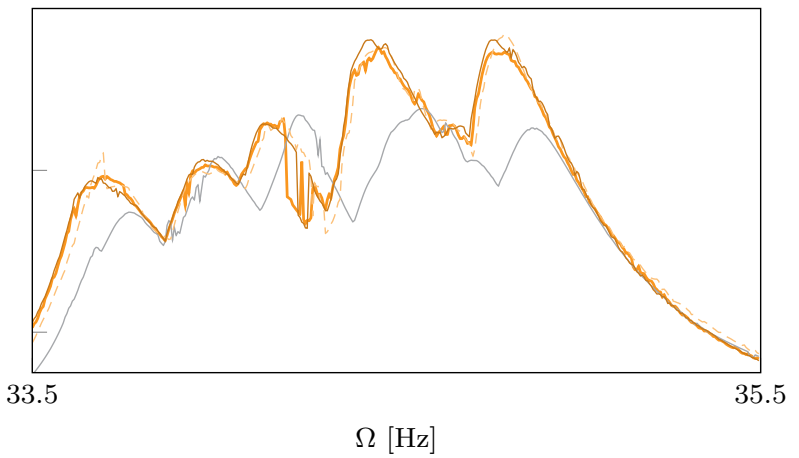

(b) space convergence: $\eta=12(-), \eta=36(=-), \eta=60$ $(-)$ and $\eta=96(-)$

Figure 9. Time and space convergences on the FRF for $n_{d}=4$

later in Section 5.

Finally, the obtained FRF for a small variation of key parameters - the casing maximal deformation and the initial clearance - are superimposed in Fig. 8. The fact that for small variations of the maximal casing deformation amplitude obtained FRF are simply slightly shifted highlights the robustness of the results.

\subsection{Time and Space Convergence}

In order to underline the convergence of the obtained results with respect to the time step $h$ of the time integration procedure, the nonlinear FRF obtained for different values of $h$ are plotted in Fig. 9a. FRF obtained for any value of $h>5 \times 10^{-5}$ s are almost perfectly superimposed. The slight differences that appear in the previously identified area for $\Omega \in[34.2,34.3] \mathrm{Hz}$ further suggest the co-existence of two stable branches of solution, as the variation of $h$ causes a small variation in the detection of the initial contact. In the following, all simulations are run considering $h=10^{-6} \mathrm{~s}$.

Space convergence is checked by varying the reduction parameter $\eta$ of the ROM. FRF obtained for different values of $\eta$, plotted in Fig. 9b, are almost perfectly superimposed from $\eta>36$. In the following, $\eta=60$ is considered so that the ROM contains a total of 84 dof: 24 boundary dof for contact management and 60 modal dof. 


\section{Nonlinear Stochastic Analysis}

Stochastic results depicting mistuning amplification for contact simulations are presented and discussed in this section. Contact simulation parameters are recalled in Tab. 4 . After a careful assessment of the stochastic result convergence, predicted nonlinear and linear amplifications are compared for different values of $n_{d}$ and mistuning levels. In particular, a subsection is dedicated to the configuration $n_{d}=4$ with a cross-analysis based on all the mistuning patterns used for contact simulations. Finally, the influence of structural damping on predicted amplifications is assessed.

Table 4. Contact simulation parameters.

\begin{aligned} & \hline time step $h=1 \cdot 10^{-6} \mathrm{~s} \\ &$\hline number of modal dof $\eta=60 \\ &$\hline total number of dof 84 \\ & \hline casing initial clearance $0.25 \mathrm{~mm} \\ &$\hline blade/casing maximal deformation $1.25 \mathrm{~mm} \\ &$\hline\end{aligned}

\subsection{Convergence}

Due to the nonlinear nature of each contact simulation, there is no empirical guideline on how many samples are required for the characterization of the vibration amplification using Monte Carlo simulations. On the one hand, it is a prerequisite to reach convergence on the percentile values so that presented results are relevant, but on the other hand the high cost of these nonlinear simulations in terms of computation times (the nonlinear FRF for each sample requires about one hour calculation on a standard i7 processor based PC) makes it difficult to reach the number of samples usually considered in a linear and less expensive context [1]. Accordingly, convergence on the percentile values is here carefully assessed both qualitatively and quantitatively in order to ensure efficient Monte Carlo simulations.

\subsubsection{Qualitative Assessment}

Qualitative convergence is checked through the stabilization of the mean of amplification with regards to the number of considered samples, for each percentile and each mistuning level $\sigma$, as shown in Fig. 10. Semi-log scales are used to emphasize any variation of the results, thus avoiding overestimating convergence rates. It is observed that convergence is reached for 2,000 samples per mistuning level for $n_{d}=6$ while 3,000 samples are required for $n_{d}=3$ and 5. Finally, 4,000 samples must be considered for $n_{d}=4$.

\subsubsection{Quantitative Assessment}

Quantitative convergence is checked using the law of large numbers [37] and the central limit theorem [37] on the standard error of the mean. These tools allow for the definition of a convergence error:

$\operatorname{error}\left(p_{s}\right)=Z \sqrt{\frac{V\left(p_{s}\right)}{s}}$

where $p_{s}$ represents the value obtained for the percentile considered, calculated on $s$ samples with a given step (set here to 10 samples), $V$ is the variance, thus making $\sqrt{\frac{V\left(p_{s}\right)}{s}}$ the standard error of the mean, and $Z$ is the confidence coefficient determined with the right-hand side standard normal Z-table. Classically, confidence level values used are $95 \%, 99 \%$ and $99.5 \%$ [37]. In this study, a $99.5 \%$ confidence level is considered to ensure high accuracy results, 


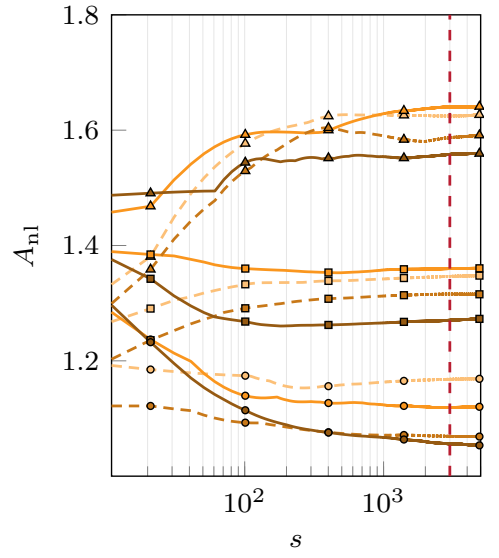

(a) $n_{d}=3$

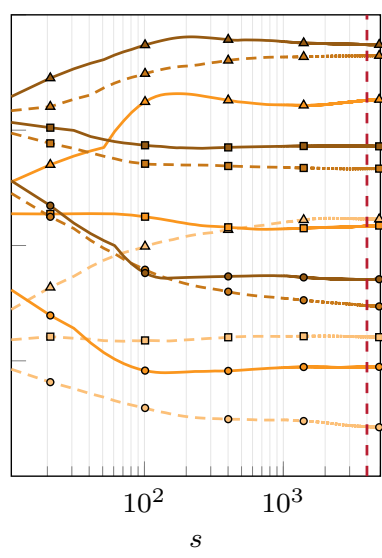

(b) $n_{d}=4$

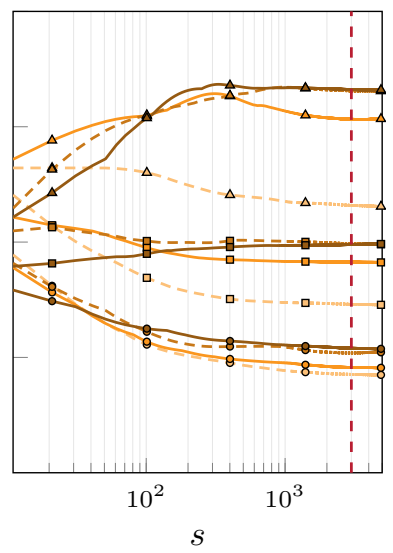

(c) $n_{d}=5$

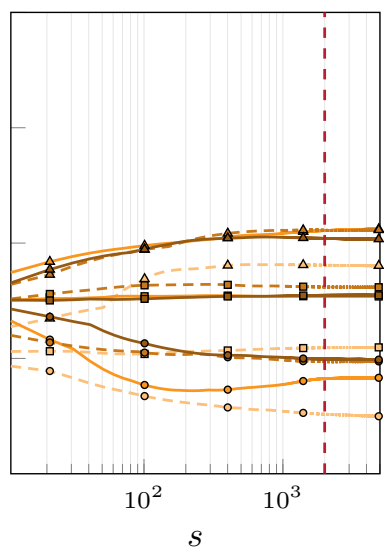

(d) $n_{d}=6$

Figure 10. Qualitative convergence assessment on percentile 1 ( ○), 50 ( $\square$ ) and 99 ( $\Delta$ ) for $\sigma=0.6 \%(==)$, $1.2 \%(-)$ ), $1.7 \%(-=)$ and $2.3 \%(-)$

hence $Z=2.81$. The variance is estimated with its unbiased estimator [37]:

$V\left(p_{s}\right)=\frac{1}{s-1} \sum_{i=1}^{s}\left|p_{i}-\bar{p}_{s}\right|^{2}$

where $p_{i}$ is the percentile value for $i$ samples and $\bar{p}_{s}$ is the mean of $p_{s}$. It should be noted that the defined error advantageously does not depend on the mean of all the samples. Convergence errors with regards to the number of samples for various nodal diameters $n_{d}$ are depicted in Fig. 11. It is assumed that results are converged for error $\left(p_{s}\right)<0.5 \%$. Monte Carlo simulations for $n_{d}=6$ are the fastest to converge, with a convergence criteria reached as early as 500 samples per mistuning level, while simulations for $n_{d}=3$ require a minimum of 2,000 samples.

\subsubsection{Global Assessment}

Both quantitative and qualitative analyses of the carried out stochastic simulations underline that, in the worst configuration, reaching convergence requires 4,000 samples. Interestingly, it thus seems that the contact constraints imposed on the bladed disk allow for a faster stochastic convergence than what is found in the literature for linear investigations where around 10,000 samples are usually considered [1], a value that is indeed found to yield convergence for linear simulations presented in section 2.3.2. This may be a consequence of the reduced admissible domain for vibrations in the radial direction due to contact. In order to ensure fully converged results, 5,000 samples are used in this study for each point $\left(n_{d}, \sigma\right)$ of the investigated parameter space.

\subsection{Vibration Amplification}

\subsubsection{Comparison to Linear Results}

Linear and nonlinear amplification factors (denoted respectively $A_{\text {lin }}$ and $A_{\mathrm{n} 1}$ ) for various $n_{d}$ and $\sigma$ are drawn in Fig. 12. The comparative analysis of these two types of amplification highlight the strong influence of both $n_{d}$ and $\sigma$. Indeed, while nonlinear amplifications are overall much higher than linear amplifications (see $n_{d}=3,4$ and 5), it is noticeable that nonlinear amplifications are predicted below linear amplifications for $n_{d}=6$. With respect to $\sigma$, the gap between $A_{\text {lin }}$ and $A_{\mathrm{nl}}$ is maximal for lower $\sigma$ for $n_{d}=3$ and 5 while it monotonically increases with $\sigma$ for $n_{d}=4$ and 6 . It is worth noting that these trends are consistent throughout percentiles.

Nonlinear amplifications are the largest for $n_{d}=4$ : they are plotted in Fig. $12 \mathrm{~d}$ for all percentiles along corresponding linear amplifications. Percentile 50 for nonlinear amplifications is almost superimposed with percentile 99 for linear amplifications, and becomes even higher for $\sigma=2.3 \%$. These results suggest a much higher 


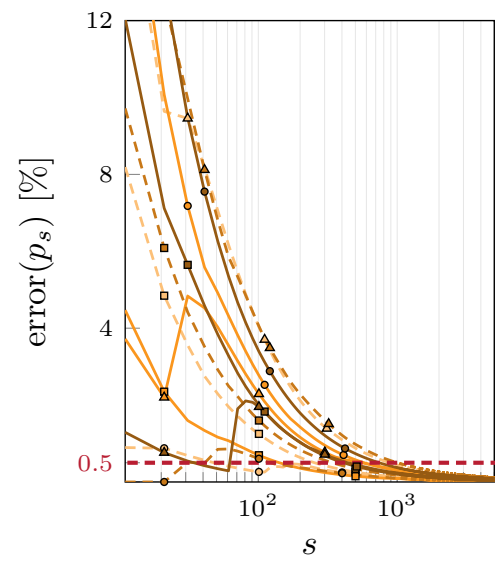

(a) $n_{d}=3$

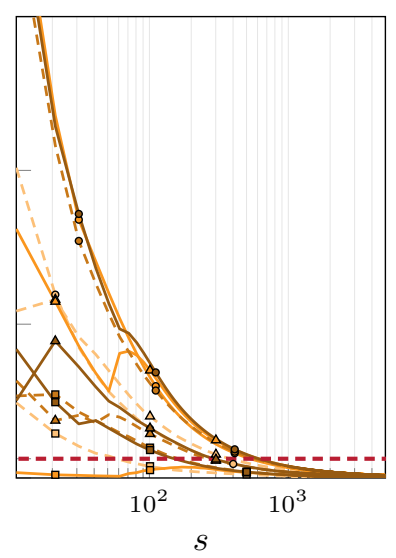

(b) $n_{d}=4$

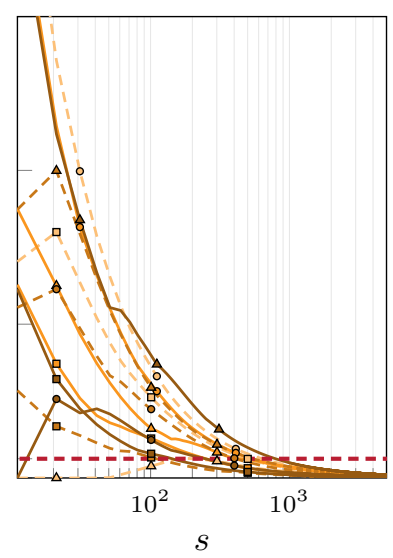

(c) $n_{d}=5$

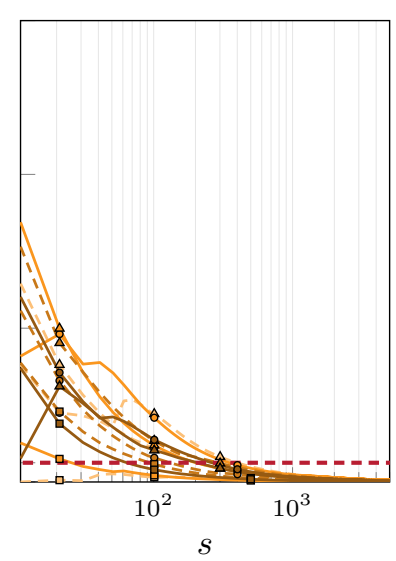

(d) $n_{d}=6$

Figure 11. Quantitative convergence assessment on percentile 1 ( ○), 50 ( $\square$ ) and $99(\Delta)$ for $\sigma=0.6 \%(=-)$, $1.2 \%(-)$, $1.7 \%(--)$ and $2.3 \%(-)$

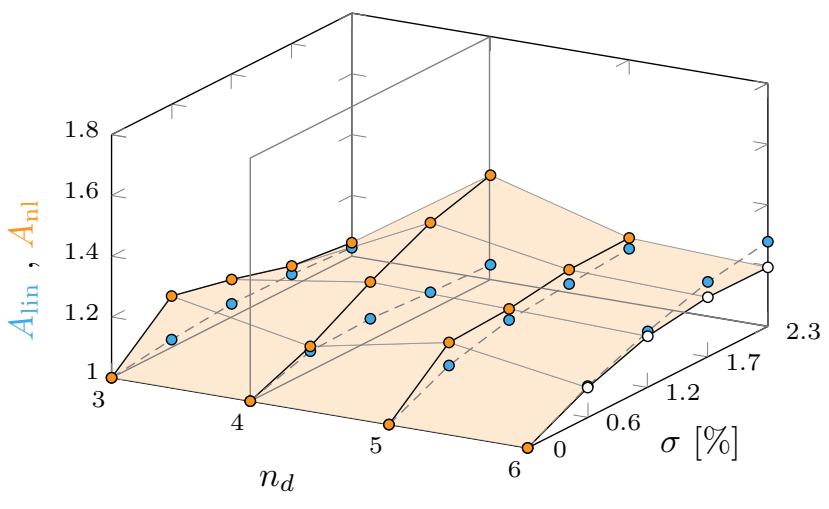

(a) percentile 1

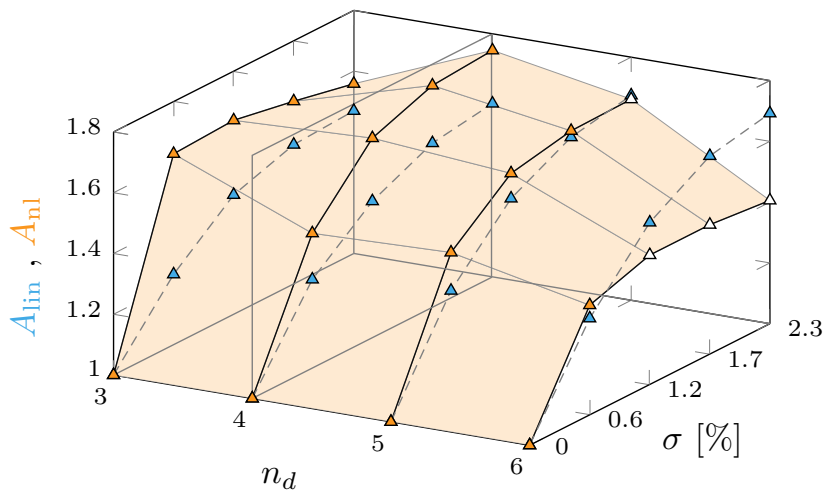

(c) percentile 99

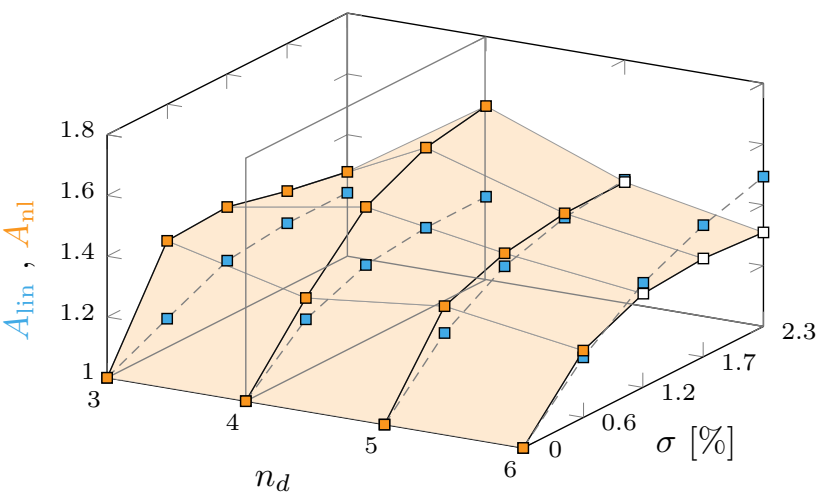

(b) percentile 50

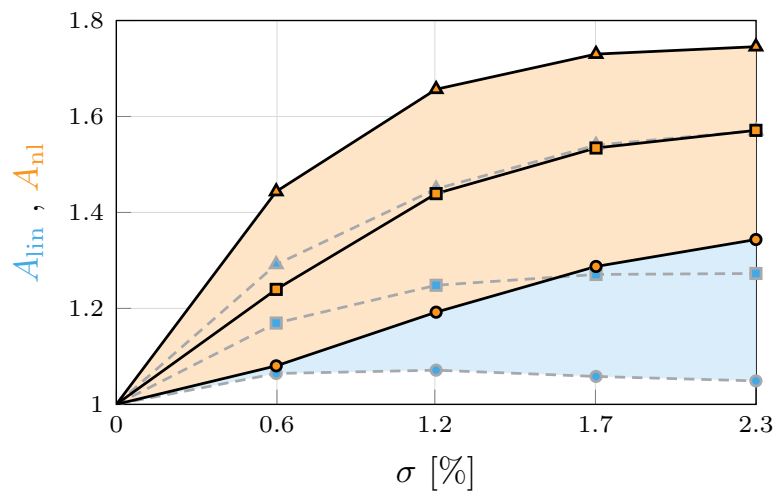

(d) all percentiles for $n_{d}=4$

Figure 12. Nonlinear $(\square)$ and linear $(\square)$ amplification for different $n_{d}$, with percentiles $1(\circ), 50(\square)$ and 99 ( $\triangle$ ) 


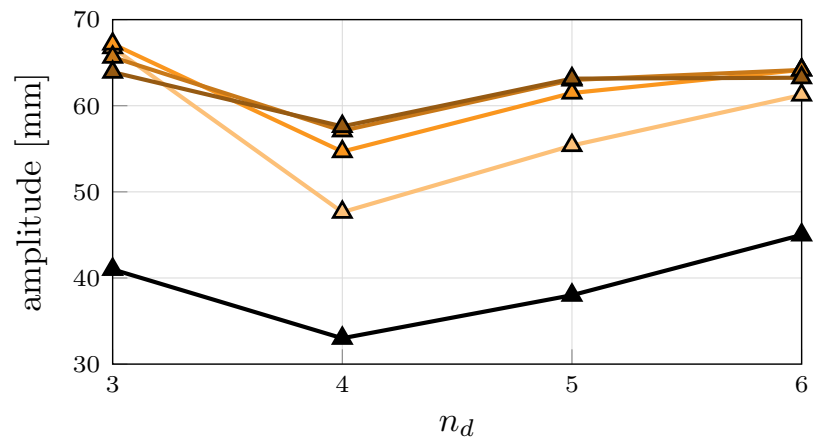

Figure 13. Maximal amplitudes of vibration for the tuned ( $\boldsymbol{-}-$ ) and mistuned bladed disks (percentile 99$)$ for $\sigma=0.6 \%(\boldsymbol{\Delta})$, $\sigma=1.2 \%(\boldsymbol{\Delta}), \sigma=1.7 \%(\boldsymbol{\Delta})$ and $\sigma=2.3 \%(\boldsymbol{\Delta})$ during contact simulations

sensitivity of the bladed disk model to mistuning when contact events occur. Maximum amplifications in a linear framework ( $57 \%$ for percentile 99 , as $A_{\text {lin }}=1.57$ ) are indeed significantly increased when structural contacts occur (75\% for percentile 99 , as $A_{\mathrm{nl}}=1.75$ ).

These results also underline that numerical predictions made on the vibration behavior of a tuned bladed disk undergoing structural contacts are robust with respect to small mistuning. Thus, interactions identified for the tuned case are also interactions for mistuned bladed disks with potentially much higher amplitudes of vibration.

\subsubsection{Analysis with Regards to the Displacements}

The linear amplification $A_{\text {lin }}$ variation with respect to $n_{d}$ is explained mostly in two ways in the literature: (1) the forcing frequency may correspond to a veering region [32,38] or, (2) the forcing frequency may excite closely spaced blade dominated modes, identified on the tuned system [33]. As underlined in the SAFE diagram, see Fig. 2, the proposed model does not feature veering regions around the forcing frequency (corresponding to the 1B modal family), but modes of the $1 \mathrm{~B}$ family are indeed closely spaced.

Yet, to the best of the authors' knowledge, there is no mention of the influence of $n_{d}$ on nonlinear amplifications in the literature. In order to explain the observations made in the previous section, it is proposed to put them into perspective in light of the maximal amplitudes of vibration given in Fig. 13. For all degrees of mistuning $\sigma$, amplitudes of vibration strongly increase when looking at percentiles 99 of the stochastic results. However, it is noticeable that for $n_{d}=3$ and $n_{d}=6$, for which tuned amplitudes of vibration are the highest, mistuned amplitudes of vibrations are clustered within a very narrow range located around $65 \mathrm{~mm}$.

Conversely, values of $n_{d}$ for which the tuned system features lowest amplitudes of vibration $\left(n_{d}=4\right.$ and $\left.n_{d}=5\right)$ yield similar trends as increasing mistuning degree leads to higher amplitudes of vibration. Contrary to the linear configuration where there is no constraint on the bladed disk vibration, contact with the surrounding casing does constrain the bladed disks amplitudes of vibration. Thus, it appears that clustered results predicted for $n_{d}=3$ and $n_{d}=6$ are essentially due to an over-constrained domain of vibration: mistuning amplification is here mitigated by the rigid casing.

In that sense, the proposed computation of amplifications as a ratio of displacements at the blade-tips may provide an optimistic representation of what is really happening for $n_{d}=3$ and $n_{d}=6$. In order to get further insight on amplification ratios, a nonlinear amplification factor based on blade strain energies and thus using the displacement of all the blade dof is proposed in the next section.

\subsubsection{Analysis with Regards to the Strain Energy}

The strain energy $\mathcal{E}$ of a finite element system can be calculated with the generalized displacements vectors and the stiffness matrix. In the reduced model used in this study, the strain energy of each blade is computed by projecting the displacements calculated for the ROM onto the physical basis. Similarly to the maximal amplitude, strain energies at a given angular speed are calculated over the last 3 revolutions, for each blade $i$. The stochastic quantity of interest is defined as the nonlinear energy amplification $A_{\mathrm{nl}}(\mathcal{E})$, computed as the ratio between the maximum 
strain energy of the mistuned model and the maximum strain energy of the tuned model.

Two sample sets from section 4 are considered: (1) the 100 samples with the maximal displacement amplification $\left(A_{\mathrm{nl}}\right)$ for the contact simulations for $n_{d}=4$ and (2) the 100 samples with the maximal displacement amplification for $n_{d}=6$. The corresponding mistuning levels for these sets are between $1.2 \%$ and $2.3 \%$. The energy amplification $A_{\mathrm{nl}}(\mathcal{E})$ is computed for these samples in the same contact configurations, $n_{d}=4$ and $n_{d}=6$. Results are presented in Fig. 14, where the black curves delimit the results for all samples.

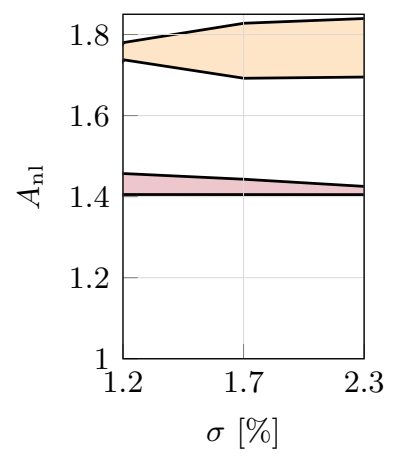

(a) displacement amplification

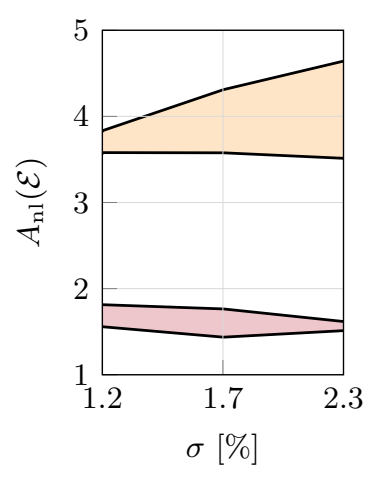

(b) energy amplification

Figure 14. Amplifications for 100 samples, for $n_{d}=4(\square)$ and $n_{d}=6(\square)$

In Fig. 14a, samples for $n_{d}=4$ present a greater $A_{\mathrm{nl}}$ than samples for $n_{d}=6$, which is consistent with the results on percentile 99 presented in Fig. 12. Results for $A_{\mathrm{nl}}(\mathcal{E})$, plotted in Fig. 14b, show an even greater gap between $n_{d}=4$ and $n_{d}=6$, with $n_{d}=4$ still presenting higher amplifications. In the light of these results, $n_{d}=6$ contact configuration does not yield a greater $A_{\mathrm{nl}}(\mathcal{E})$. Yet, it should be noted that the small amount of samples available for this analysis does not allow stochastically significant results, and the samples used may induce a selection bias as they have been selected on their value for $A_{\mathrm{nl}}$. It is also found that results for $n_{d}=4$ present a higher dispersion than those for $n_{d}=6$, which is magnified for $A_{\mathrm{nl}}(\mathcal{E})$. As was the case for the maximum amplitudes, this can be explained by the higher number of contact areas increasing the solicitation frequency and limiting the space in which the blades can vibrate. Lastly, it can be noted that the order of magnitude for the displacement and the energy amplifications are very different.

In the end, $A_{\mathrm{nl}}(\mathcal{E})$ results confirm $A_{\mathrm{nl}}$ results, where $n_{d}=4$ is found to provide the most representative results: as predicted amplitudes of vibration for the tuned case are lower, it is possible to fully capture their increase due to mistuning. For this reason, the focus is made on the configuration $n_{d}=4$ in the following.

\subsection{Cross-Analysis for $n_{d}=4$}

The 5,000 samples considered for contact simulations with $n_{d}=4$ and for each mistuning level $\sigma$ are subjected to the linear forcing defined in Eq. (5). Their amplifications are thus obtained in both linear and nonlinear contexts. These values are depicted in the $\left(A_{\mathrm{nl}}, A_{\text {lin }}\right)$ plane, see Fig. 15. Cumulative densities, calculated from the highest to the lowest density regions, are preferred to a scatter plot in order to get a clearer view of denser areas.

Overall, the majority of mistuning patterns features $A_{\mathrm{nl}}>A_{\operatorname{lin}}$ : the majority of data points are indeed located above the line $A_{\mathrm{nl}}=A_{\operatorname{lin}}$. This trend is reinforced as the mistuning level $\sigma$ increases. More precisely, for $\sigma=0.6 \%$, the nonlinear amplification is higher than the linear amplification for $82 \%$ of the samples. This number grows to $96 \%$ for $\sigma=2.3 \%$. It is noticeable that mistuning patterns with highest values of $A_{\mathrm{nl}}$ spread along a wide range of $A_{\text {lin }}$ values. For instance, for $\sigma=1.7 \%$, mistuning patterns above percentile 99 of $A_{\mathrm{nl}}$ values correspond to $A_{\text {lin }} \in[1,1.7]$. These results thus underline that there is no correlation between mistuning patterns featuring high linear amplifications and mistuning patterns featuring high nonlinear amplifications. Accordingly, mitigating 


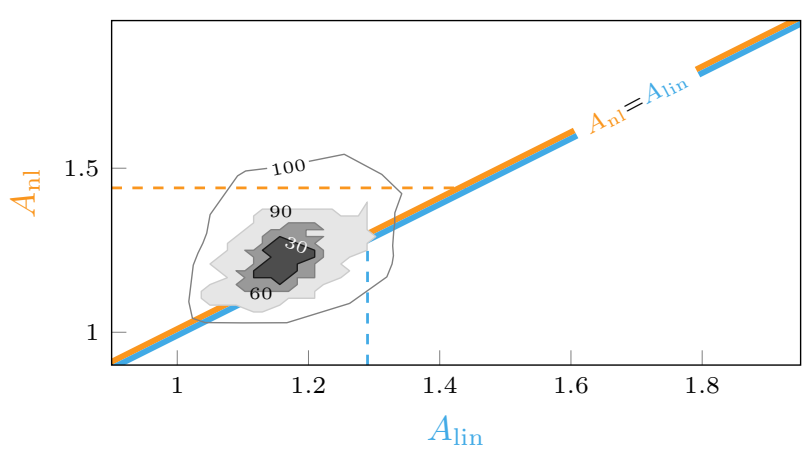

(a) $\sigma=0.6 \%$

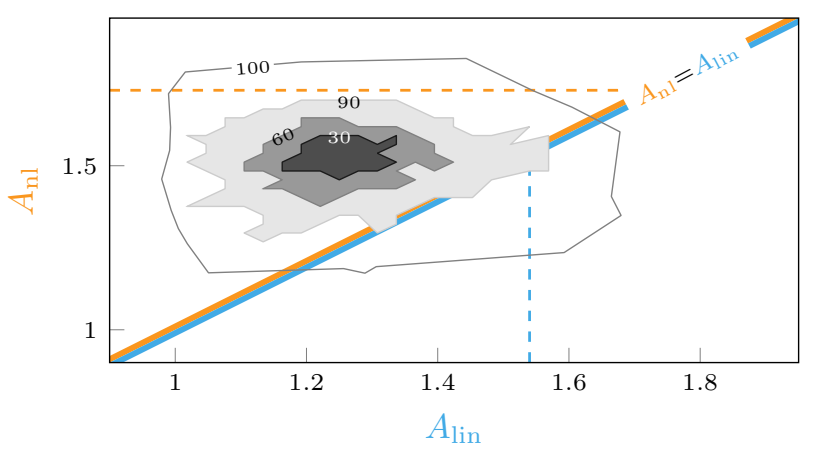

(c) $\sigma=1.7 \%$

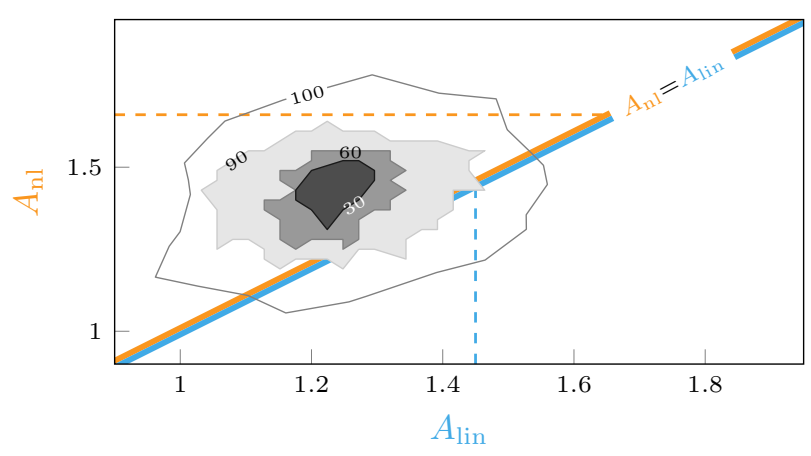

(b) $\sigma=1.2 \%$

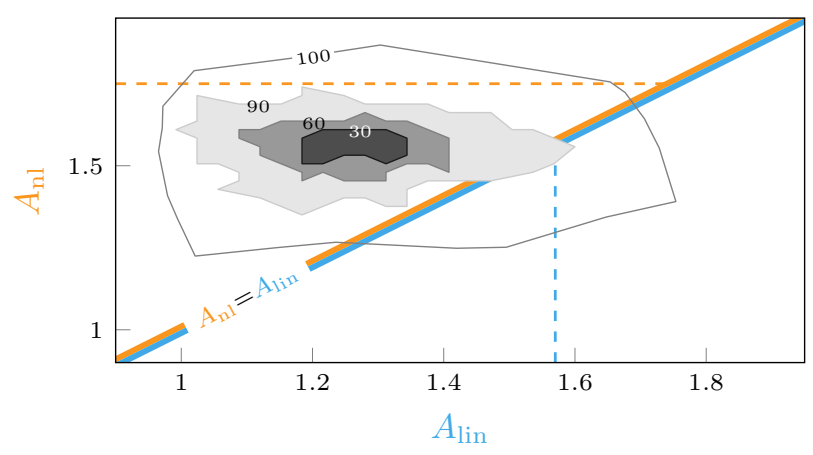

(d) $\sigma=2.3 \%$

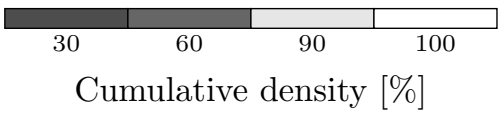

Figure 15. Cross-analysis of mistuning amplification, for $n_{d}=4$, with percentiles 99 for nonlinear $(=-)$ and linear $(=-)$ simulations

linear amplifications and nonlinear amplifications may call for distinct design strategies and existing techniques available in the literature $[8,9,10]$ should also be assessed in a structurally nonlinear context.

\subsection{Influence of Damping}

Next generation designs for bladed disk typically include integrally bladed disks or blisks. They are advantageously lighter but also more prone to high amplitudes of vibration due to a lower structural damping. Blisks have been lately studied both numerically [28] and experimentally $[39,40]$ in a linear context and under aerodynamic flutter. This section intends to give insight on the effect of lower structural damping on nonlinear amplifications.

Stochastic simulations have been performed using the same methodology as previously described for a modal damping ratio of $4 \cdot 10^{-3}$, corresponding to a $20 \%$ damping reduction. Amplification diagrams for linear and contact simulations are given in Fig. 16. It is remarkable that linear amplifications are only slightly impacted by the lower structural damping. Results, presented only for $n_{d}=4$ for the sake of clarity, are consistent with those obtained for other $n_{d}$.

On the contrary, all percentiles of nonlinear amplifications are significantly shifted up: nonlinear amplification for $\sigma=2.3 \%$ at percentile 99 goes from $75 \%$ to more than $84 \%$. With a lower damping ratio, these results predict that contact occurrences could thus result in even more detrimental vibration amplitudes. 


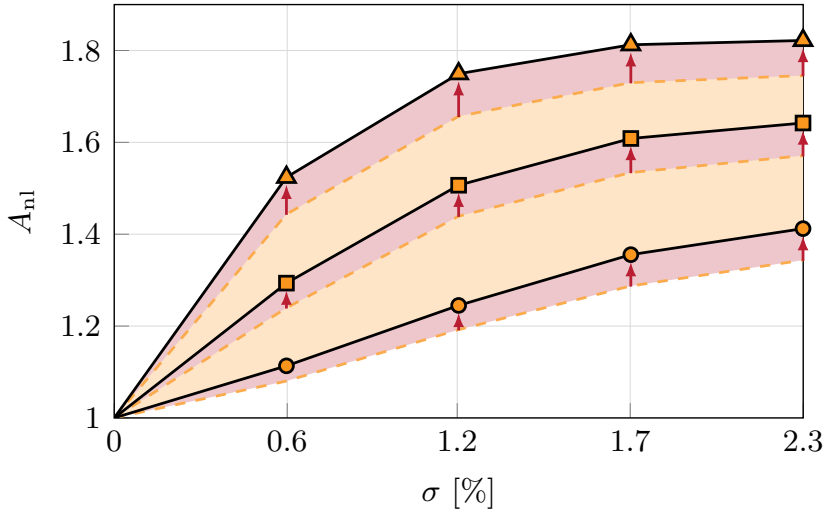

(a) linear simulations

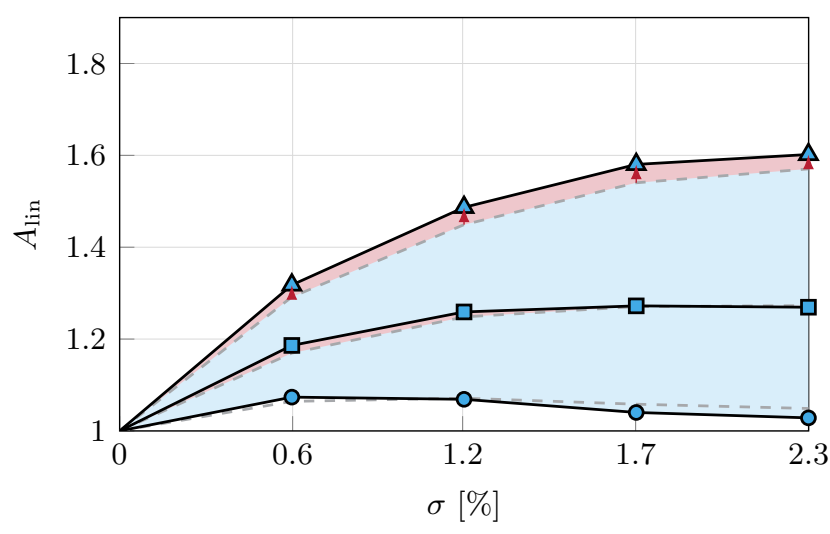

(b) contact simulations

Figure 16. Amplification variation $(\longrightarrow)$ between a modal damping ratio of $5 \times 10^{-3}(\boldsymbol{-}-\boldsymbol{)})$ and of $4 \times 10^{-3}(\boldsymbol{-})$, for $n_{d}=4$

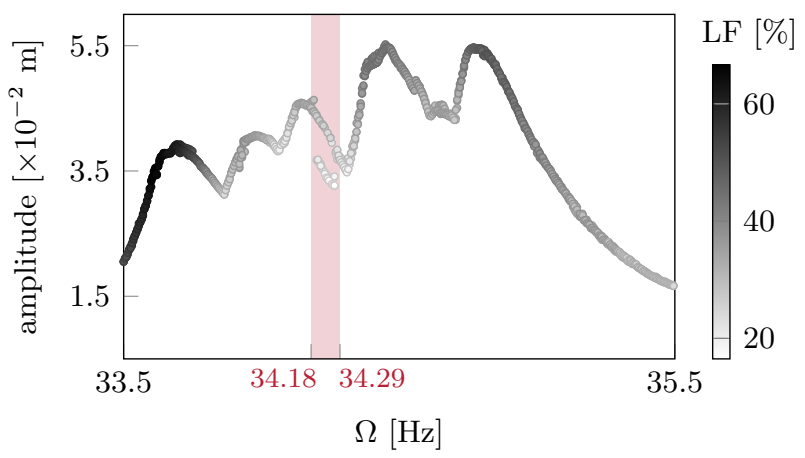

(a) FRF

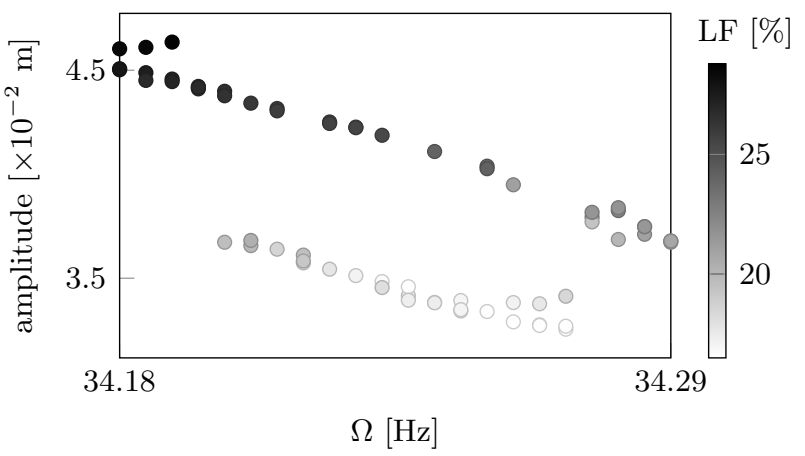

(b) zoom

Figure 17. Localization factor for each point of the FRF

\section{Evidence of Co-Existing Stable Solutions}

In this section, additional details are provided with respect to the co-existing solutions identified in Fig. 9a for various times steps (from $h=5 \times 10^{-7} \mathrm{~s}$ to $h=5 \times 10^{-5} \mathrm{~s}$ ). A finer resolution in angular speed is adopted: $\delta \Omega=0.005 \mathrm{~Hz}$. Results are plotted in Fig. 17. In addition to the amplitude of the steady state, a color code is employed depending on the value of a global criterion used to characterize the bladed disk vibration behavior, with white being its lowest value and black its highest.

Within the context of the analysis of mistuned bladed disk, the selected criterion is based on the localization factor defined in [28] for free vibrations. The root mean square of the maximal vibration amplitude at the blade-tips, $\mathbf{U}$, is calculated with:

$\operatorname{RMS}(\mathbf{U})=\sqrt{\frac{1}{N} \sum_{i=1}^{N}(U(i))^{2}} \quad, \quad i \in[1, N]$

with $N$ the number of blades. For non-localized forced vibrations, all blades display the same maximal vibration amplitude, hence $\operatorname{RMS}(\mathbf{U})=\max (\mathbf{U})$. Conversely, for extremely localized vibrations only one blade vibrates, 
implying $\operatorname{RMS}(\mathbf{U})=\frac{\max (\mathbf{U})}{\sqrt{N}}$. The following ratio:

$\zeta=\frac{\max (\mathbf{U})}{\operatorname{RMS}(\mathbf{U})}$

thus varies from 1 to $\sqrt{N}$. The localization factor LF, varying from $0 \%$ to $100 \%$, is thus defined as:

$\mathrm{LF}=\frac{\zeta-1}{\sqrt{N}-1} \times 100$

The localization factor is calculated for each point of the nonlinear FRF depicted in Fig. 17. Solutions over $\Omega \in[33.5,35.5] \mathrm{Hz}$ in Fig. 17a exhibit a localization factor from $17 \%$ to $65 \%$. Localization appears to change smoothly with the angular speed, except in the range $[34.18,34.29] \mathrm{Hz}$. Over this angular speed range, where a zoom is made in Fig. 17b, it is noticeable from the different gray shades that each branch of solutions feature very distinct values of the localization factor: the upper branch exhibit higher localization factors (as indicated by its darker dots), with a mean of $25.9 \%$, when the lower branch has a localization factor of $17.4 \%$ on average. While more in-depth analyses are required to further understand the link between localization and resonances or even potential bifurcations, these results confirm the intrinsic differences between solutions found on each branch of solution, suggesting distinct vibration behaviors.

Considering the global FRF in Fig. 17a, the gray scale shows that there is no correlation between the localization factor on the bladed disk and the maximal amplitude at the blade-tips. Indeed, LF is found maximum (67.9\%) for $\Omega=33.66 \mathrm{~Hz}$, where the amplitude at the blade-tip is of $38.9 \mathrm{~mm}$, but $L F=49.0 \%$ for $\Omega=34.78 \mathrm{~Hz}$, where the amplitude is maximum $(58.1 \mathrm{~mm})$. Finally, the highest localization factors for this FRF are found before the first resonance.

\section{Conclusion}

The influence of small mistuning on the amplitudes of vibration of a 2D phenomenological bladed disk following blade-tip/casing contact events is assessed in this paper. The carried out analysis relies on a numerical procedure dedicated to the prediction of blade-tip/casing contact induced interactions that has been previously validated with respect to both experimental set-ups and academic test cases. It is based on numerical time integration with a Lagrange multiplier-based contact algorithm. Mistuning is accounted for as a variation of the blades Young's modulus. Attention is paid to the validation of the proposed numerical strategy: its convergence with respect to key parameters is carefully checked. It is also underlined that presented results are robust with respect to simulation parameters including the casing deformation amplitude and the blade-tip/casing clearance.

By means of Monte Carlo simulations, linear and nonlinear amplifications-defined as the ratio between the maximal amplitude of vibration of mistuned structures and the one of the tuned model—are analyzed comparatively for different configurations. In comparison to amplifications predicted in a linear context, presented results suggest that mistuning may lead to even higher amplifications when contacts are accounted for. These results are observed for various configurations and several mistuning levels, and are consistent with amplifications related to the strain energy. Also, this study confirms that predicted interactions on a tuned bladed disk are robust with respect to small mistuning: all predicted critical speeds on a tuned blade disk are also found critical for mistuned structures.

The carried out cross-analysis of linear and nonlinear amplifications for a given contact configuration underlines that there is no correlation between mistuning patterns exhibiting high amplifications in a context or another. Indeed, the highest nonlinear amplifications can be predicted for mistuned patterns featuring low to high linear amplifications. Results obtained with this phenomenological bladed disk thus hint that design strategies to mitigate mistuning amplifications in a linear context may not be well suited for mitigating nonlinear amplifications. Existing techniques available in the literature to mitigate linear amplifications $[8,9,10]$ should then be assessed in a structurally nonlinear context. Furthermore, stochastic simulations have been carried out with a damping ratio lowered by $20 \%$. It was found that mistuning amplification in nonlinear simulations could be even more detrimental than the corresponding amplification in linear simulations for lowly damped systems, such as blisks. 
By means of a localization factor, differences in the vibration behavior are highlighted and enable to clearly identify co-existing stable solutions. It has thus been showed that the academic ROM used in this study, as well as the contact algorithm relying on time integration, can capture complex dynamic behaviors.

Finally, presented results suggest that the severity of contact events may be drastically magnified in presence of mistuning. Studying the influence of mistuning in a nonlinear context would then be of crucial to ensure the safe operation of bladed disks.

\section{Acknowledgment}

This research was supported by the Fonds de recherche du Québec - Nature et technologies (FRQ-NT) and the Natural Sciences and Engineering Research Council of Canada (NSERC).

\section{References}

[1] M. P. Castanier, C. Pierre, Modeling and Analysis of Mistuned Bladed Disk Vibration: Status and Emerging Directions, Journal of Propulsion and Power 22 (2) (2006) 384-396. do i : 10.2514/1. 16345.

[2] D. J. Ewins, The effects of detuning upon the forced vibrations of bladed disks, Journal of Sound and Vibration 9 (1) (1969) 65-79. do i : 10 . 1016/0022-460X (69)90264-8.

[3] R. C. F. Dye, T. A. Henry, Vibration Amplitudes of Compressor Blades Resulting From Scatter in Blade Natural Frequencies, Journal of Engineering for Power 91 (3) (1969) 182-187. do i : 10.1115/1.3574726.

[4] C. Pierre, P. D. Cha, Strong Mode Localization in Nearly Periodic Disordered Structures, AIAA Journal 27 (2) (1989) 227-241. doi:10.2514/3.10085.

[5] R. Bladh, M. P. Castanier, C. Pierre, Reduced Order Modeling and Vibration Analysis of Mistuned Bladed Disk Assemblies With Shrouds, Journal of Engineering for Gas Turbines and Power 121 (3) (1999) 515-522. do i: 10.1115/1.2818503.

[6] Y.-J. Chan, D. J. Ewins, Management of the variability of vibration response levels in mistuned bladed discs using robust design concepts. Part 1: Parameter design, Mechanical Systems and Signal Processing 24 (8) (2010) 2777-2791. do i : 10.1016/ j .ymssp.2010.06.006.

[7] Y.-J. Chan, D. J. Ewins, Management of the variability of vibration response levels in mistuned bladed discs using robust design concepts. Part 2: Tolerance design, Mechanical Systems and Signal Processing 24 (8) (2010) 2792-2806. do i:10.1016/ j ymssp.2010.06.005.

[8] M. P. Castanier, C. Pierre, Using Intentional Mistuning in the Design of Turbomachinery Rotors, AIAA Journal 40 (10) (2002) 2077-2086. do i : 10.2514/3. 15298.

[9] Y. Han, R. Murthy, M. P. Mignolet, J. Lentz, Optimization of Intentional Mistuning Patterns for the Mitigation of the Effects of Random Mistuning, Journal of Engineering for Gas Turbines and Power 136 (6) (2014) 1-9. doi: $10.1115 / 1.4026141$.

[10] B. Beirow, F. Figaschewsky, A. Kühhorn, A. Bornhorn, Vibration analysis of an axial turbine blisk with optimized intentional mistuning pattern, Journal of Sound and Vibration 442 (3) (2019) 11-27. do i: 10.1016/ j.jsv.2018.10.064.

[11] F. Figaschewsky, A. Kühhorn, B. Beirow, J. Nipkau, T. Giersch, B. Power, Design and analysis of an intentional mistuning experiment reducing flutter susceptibility and minimizing forced response of a jet engine fan, in: ASME Turbo Expo, 2017, pp. V07BT36A020-V07BT36A020. do i : 10.1115/GT2017-64621.

[12] R. Corral, O. Khemiri, C. Martel, Design of mistuning patterns to control the vibration amplitude of unstable rotor blades, Aerospace Science and Technology 80 (2018) 20-28. do i : 10.1016/ j . ast.2018.06.034.

[13] C. Joannin, B. Chouvion, F. Thouverez, J.-P. Ousty, M. Mbaye, A nonlinear component mode synthesis method for the computation of steady-state vibrations in non-conservative systems, Mechanical Systems and Signal Processing 83 (15) (2017) 75-92. do i: 10.1016/ j ymssp.2016.05.044. 
[14] G. Óttarsson, C. Pierre, On the effects of interblade coupling on the statistics of maximum forced response amplitudes in mistuned bladed disks, AIAA 1494-CP (1995) 3070-3078. do i : 10 .2514/6 . 1995-1494.

[15] R. Rajasekharan, E. Petrov, Analysis of Deformation of Mistuned Bladed Disks With Friction and Random Crystal Anisotropy Orientation Using Gradient-Based Polynomial Chaos Expansion, in: ASME Turbo Expo, 2018, p. V07CT35A029. doi:10.1115/GT2018-76566.

[16] R. J. Williams, Simulation of Blade Casing Interaction Phenomena in Gas Turbines Resulting from Heavy Tip Rubs Using an Implicit Time Marching Method, in: ASME Turbo Expo, Vol. 6, 2011, pp. 1-10. doi: 10.1115/GT2011-45495.

[17] K. E. Turner, M. Dunn, C. Padova, Airfoil Deflection Characteristics During Rub Events, Journal of Turbomachinery 134 (1) (2012) 011018. do i : 10.1115/1.4003257.

[18] A. Millecamps, J.-F. Brunel, P. Dufrenoy, F. Garcin, M. Nucci, Influence of Thermal Effects During Blade-Casing Contact Experiments, in: ASME IDETC-CIE, Vol. 1, 2009, pp. 855-862. do i : 10.1115/DETC2009-86842.

[19] A. Batailly, M. Legrand, A. Millecamps, F. Garcin, Numerical-Experimental Comparison in the Simulation of Rotor/Stator Interaction Through Blade-Tip/Abradable Coating Contact, Journal of Engineering for Gas Turbines and Power 134 (8) (2012) 082504. doi:10.1115/1.4006446.

[20] M. Legrand, A. Batailly, C. Pierre, Numerical Investigation of Abradable Coating Removal in Aircraft Engines Through Plastic Constitutive Law, Journal of Computational and Nonlinear Dynamics 7 (1) (2012) 011010. do i : 10.1115/1.4004951.

[21] G. Jacquet-Richardet, M. Torkhani, P. Cartraud, F. Thouverez, T. Nouri Baranger, M. Herran, C. Gibert, S. Baguet, P. Almeida, L. Peletan, Rotor to stator contacts in turbomachines. Review and application, Mechanical Systems and Signal Processing 40 (2) (2013) 401-420. do i : 10.1016/ j ymssp. 2013.05.010.

[22] P. Almeida, C. Gibert, F. Thouverez, Numerical Analysis of Bladed Disk - Casing Contact with Friction and Wear, Journal of Engineering for Gas Turbines and Power 138 (12) (2016) 122802.

[23] M. Legrand, C. Pierre, P. Cartraud, J. P. Lombard, Two-dimensional modeling of an aircraft engine structural bladed disk-casing modal interaction, Journal of Sound and Vibration 319 (1-2) (2009) 366-391. doi: 10.1016/j . jsv.2008.06.019.

[24] A. Batailly, Q. Agrapart, A. Millecamps, J. F. Brunel, Experimental and numerical simulation of a rotor/stator interaction event localized on a single blade within an industrial high-pressure compressor, Journal of Sound and Vibration 375 (2016) 308-331. doi:10.1016/ j . jsv.2016.03.016.

[25] N. J. Carpenter, R. L. Taylor, M. G. Katona, Lagrange Constraints for Transient Finite Element Surface Contact, International Journal for Numerical Methods in Engineering 32 (1) (1991) 103-128. doi:10.1002/nme. 1620320107.

[26] E. P. Petrov, D. J. Ewins, Effects of damping and varying contact area at blade-disk joints in forced response analysis of bladed disk assemblies, Journal of turbomachinery 128 (2) (2006) 403-410. doi: 10.1115/1. 2181998.

[27] M. Legrand, A. Batailly, B. Magnain, P. Cartraud, C. Pierre, Full three-dimensional investigation of structural contact interactions in turbomachines, Journal of Sound and Vibration 331 (11) (2012) 2578-2601. doi: 10.1016/ j. jsv.2012.01.017.

[28] T. Klauke, A. Kühhorn, B. Beirow, M. Golze, Numerical Investigations of Localized Vibrations of Mistuned Blade Integrated Disks (Blisks), Journal of Turbomachinery 131 (3) (2009) 031002. do i : 10. 1115/1.2985074.

[29] N. Salvat, A. Batailly, M. Legrand, Two-dimensional modeling of unilateral contact-induced shaft precessional motions in bladed-disk/casing systems, International Journal of Non-Linear Mechanics 78 (2016) 90-104. doi:10.1016/j.ijnonlinmec.2015.10.001.

[30] R.-R. Craig, M. C. C. Bampton, Coupling of Substructures for Dynamics Analyses, AIAA Journal 6 (7) (1968) 1313. doi: $10.2514 / 3.4741$. 
[31] E. Capiez-Lernout, C. Soize, J.-P. Lombard, C. Dupont, E. Seinturier, Blade manufacturing tolerances definition for a mistuned industrial bladed disk, in: ASME Turbo Expo, Vol. 6, 2004, pp. 307-316. doi:10.1115/ GT2004-53356.

[32] A. G. Joshi, B. I. Epureanu, Reduced Order Models for Blade-To-Blade Damping Variability in Mistuned Blisks, Journal of Vibration and Acoustics 134 (2012) 051015. do i: 10.1115/1.4006880.

[33] J. Judge, C. Pierre, O. Mehmed, Experimental Investigation of Mode Localization and Forced Response Amplitude Magnification for a Mistuned Bladed Disk, Journal of Engineering for Gas Turbines and Power 123 (4) (2001) 940-950. doi : 10.1115/1.1377872.

[34] J. H. Griffin, T. M. Hoosac, Model development and statistical investigation of turbine blade mistuning, Journal of Vibration Acoustics Stress and Reliability in Design 106 (2) (1984) 204-210. do i : 10.1115/1.3269170.

[35] S. H. Strogatz, Nonlinear Dynamics And Chaos, Taylor \& Francis Inc, 1994.

[36] A. Batailly, M. Legrand, A. Millecamps, F. Garcin, Conjectural Bifurcation Analysis of the Contact-Induced Vibratory Response of an Aircraft Engine Blade, Journal of Sound and Vibration 348 (21) (2015) 239-262. doi:10.1016/j.jsv.2015.03.005.

[37] C. Graham, D. Talay, Stochastic Simulation and Monte Carlo Methods, Springer, 2013.

[38] P. Wang, L. Li, Parametric Dynamics of Mistuned Bladed Disk, in: ASME Turbo Expo, Düsseldorf, Germany, 2014, p. V07BT33A022. doi:10.1115/GT2014-26678.

[39] B. Beirow, A. Kühhorn, F. Figaschewsky, J. Nipkau, Effect of Mistuning and Damping on the Forced Response of a Compressor Blisk Rotor, in: ASME Turbo Expo, 2015, pp. 1-12. do : : 10.1115/GT2015-42036.

[40] P. Hönisch, A. Kühhorn, B. Beirow, Experimental and numerical analyses of radial turbine blisks with regard to mistuning, in: ASME Turbo Expo, Vol. 6, Vancouver (Canada), 2011, pp. 971-980. doi: 10.1115/GT2011-45359. 